\title{
A first attempt to prevent amblyopia and squint by spectacle correction of abnormal refractions from age 1 year
}

\author{
R M INGRAM, C WALKER, J M WILSON, P E ARNOLD, J LUCAS, \\ AND S DALLY
}

From Kettering and District General Hospital, Kettering, Northamptonshire

SUMMARY Spectacle correction of unusually hypermetropic refractions from age 1 year did not reduce the incidence of squint or amblyopia, nor did it lead to a reduction in the severity of residual amblyopia after subsequent occlusion.

The ability to predict which children are particularly likely to present subsequently with esotropia and/or amblyopia' is an essential precursor to exploring the possibility of preventing these conditions. However, there is no point in prediction if prevention is impossible. Many children who present with squint and/or amblyopia have hypermetropic refractions at the time they present, ${ }^{2}$ and the first step in treatment is optical correction of these refractions. Since the only method of predicting squint/amblyopia was identification of hypermetropia at age 1 year,' it seemed logical to try optical correction of abnormal refractions from that age in the hope that squint/ amblyopia might be prevented. This had been suggested before, ${ }^{3-5}$ but so far as we are aware this is the first attempt to do it.

\section{Patients and methods}

The children reported on here were drawn from two population samples: (1) Children born in an around the town of Kettering. Those born in 1974-7 were refracted after cycloplegia with $1 \%$ atropine twice daily for four days, and their refractions have already been reported. ${ }^{6}$ Those born in 1978 were refracted after cycloplegia with cyclopentolate $1 \%$. (2) Children born in the town of Rushden in the years 1975-8 who were refracted after cycloplegia with cyclopentolate $1 \%$. The refractions of those born in 1975 and 1976 have also been reported. ${ }^{7}$

Correspondence to Dr R M Ingram, Kettering and District General Hospital, Rothwell Road, Kettering, Northamptonshire NN168UZ.
PROTOCOL FOR THE TRIAL

The refractive criteria for entry into the trial were either or both of $(a)$ bilateral spherical hypermetropia of $+2.00 \mathrm{DS}$ or more; $(b)$ anisometropia of +1.00 or more $D$ sphere or cyl.

Once a child had been identified as having an abnormal refraction, the mother was told that her child had a refraction which we thought was unusually likely to lead to the appearance of squint and/or amblyopia. It was not known whether spectacle correction would prevent these conditions, but, if she wished, her child could enter a trial designed to show if this was possible. It was explained that the rules of the trial required that her child would be randomly allocated to wearing glasses or not, but that she had to decide whether she wished to enter the trial before the random decision for 'treatment' or 'no treatment' was taken. The child, treated or not treated, would then be followed up every three months until the age of $3 \frac{1}{2}$ years or soon thereafter, when it would be possible to obtain an accurate assessment of vision. If in the meantime squint was detected, conventional treatment would be given.

If a child entered the trial and was randomly allocated to wear glasses, the prescription of the glasses was based on the cycloplegic retinoscopy with 2.00 D subtracted from each meridian of each eye. Spectacles were prescribed until refraction came within normal limits, that is, $<+2.00 \mathrm{DS}$ right and left, $<+1.50 \mathrm{D}$ cyl, $<1.00 \mathrm{DS}$ or cyl anisometropia.

This protocol lacked many of the features of an ideal controlled trial, principally because the future course of events was unknown. For example, it was 
impossible to plan the number of children to be included in the trial, because it was not known what proportion of children allocated spectacles would wear them, and it was impossible to speculate on what the drop-out rate would be.

One hundred and forty-nine children did not enter the trial. Those living in Kettering were not followedup, but those in Rushden were reviewed at age $31 / 2$. Further information is available on 44 of these children. The incidence of visual defects does not differ from those included in the trial.

\section{CONDUCT OF THE TRIAL}

Every attempt was made to encourage regular review, but many attendances followed reminders sent through the post and it was impossible to achieve the ideal. Some of the children who were lost to follow-up moved from the area, but some information has been obtained from colleagues elsewhere in the UK. Others dropped out but reattended after a final recall about the age of $3 \frac{1}{2}$ years or returned spontaneously because of squint or poor performance at a school vision screening test. A final vision was recorded for 265 of the 306 children who originally entered the trial.

Recording of compliance with treatment presented difficulties. Although one could be reasonably sure when children were obviously wearing glasses or not, sometimes one could do no more than guess how much or whether treatment was being given.

The end result was to be decided on the basis of: (a) Presence or absence of esotropia/intermittent esotropia; $(b)$ presence or absence of amblyopia at the age of $3 \frac{1}{2}$. This has not proved possible in practice.
ASSESSMENT AND RECORDING OF RESULTS

Our experience since this trial started in January 1975 made it necessary to change the original plan for presenting the end results. The reasons for this were as follows. (1) Uncertainty about the visual acuity of young children, particularly around the age of $31 / 2 .{ }^{8}$ (2) Uncertainty about making a definite diagnosis of amblyopia, particularly if a child had been given occlusion before visual acuity was known and acuity subsequently became normal. (3) Uncertainty about compliance with treatment. This could be ignored if it is accepted that the correct purpose of the trial should have been to assess the effect of prescribing spectacle treatment. The results of the 'treatment' group have been subdivided into two groups, namely, $\mathrm{T}+$ and $\mathrm{T} \pm / 0$ where there were doubts about whether treatment was being given or not. (4) The amount of meridional hypermetropia' had been identified as a better predictive indicator than bilateral spherical hypermetropia and/or anisometropia (meridional hypermetropia is the amount of hypermetropia in the most hypermetropic meridian).

Therefore the end result has been recorded as the last known visual acuity of the eye with lower acuity, with spectacle correction if necessary, and after occlusion had been given and often some time after occlusion had stopped.

The two groups of children (those screened after atropine and those screened after cyclopentolate) have been pooled after allowance was made for the (approximate) $0.5 \mathrm{D}^{7}$ difference between a refraction after atropine and after cyclopentolate. The refraction recorded represents the cyclopentolate equivalent of the highest amount of meridional hypermetropia at age 1 year.

Table 1 Children with $<+3 \cdot 50$ D at age 1 year

\begin{tabular}{|c|c|c|c|c|c|c|c|c|c|}
\hline & & $6 / 6$ & $6 / 9$ & $6 / 12$ & $6 / 18$ & $6 / 24$ & $6 / 36$ & $6 / 60$ or less & Unknown \\
\hline \multirow[t]{2}{*}{$\overline{T+}$} & No Squint & $44(1)$ & 12 & 1 & 1 & - & 1 & - & 2 \\
\hline & Squint & $4(1)$ & - & - & - & - & - & - & - \\
\hline & Squint & $1(1)$ & - & - & - & - & - & - & - \\
\hline \multirow[t]{2}{*}{ NT } & No Squint & 51 & $31(1)$ & - & - & - & - & - & 13 \\
\hline & Squint & $6(1)$ & $4(3)$ & $1(1)$ & $1(1)$ & - & $1(1)$ & - & - \\
\hline
\end{tabular}

Figures in parentheses show numbers of children who had occlusion.

Table 2 Children with $+3 \cdot 50$ or more Dat age 1 year

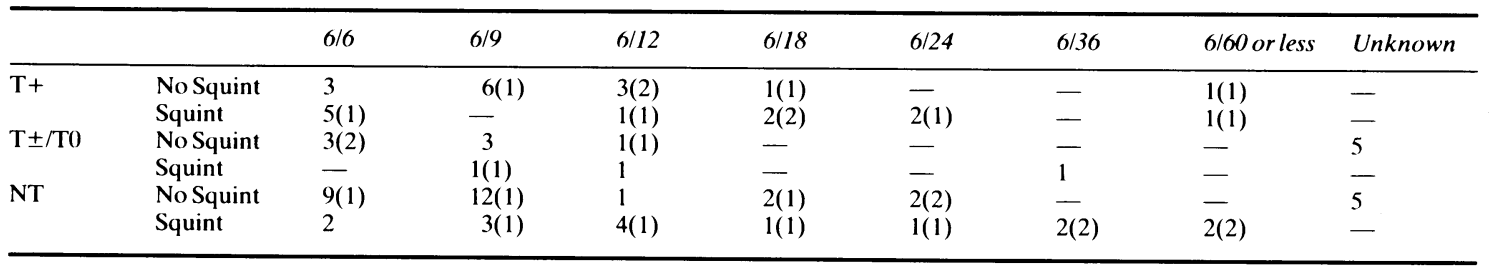

Figures in parentheses show numbers of children who had occlusion. 
Table 3 Incidence of squint

\begin{tabular}{llcr}
\hline & $T+$ & $T \pm / 0$ & $N T$ \\
\hline No squint & 73 & 37 & 108 \\
Squint & 15 & 4 & 28 \\
\hline
\end{tabular}

\section{Results}

Tables 1 and 2 summarise the findings, indicating the refraction at age 1 year (more or less than $+3.50 \mathrm{D}$ meridional hypermetropia) and the last known visual acuity, the presence of squint, and whether occlusion had been given. Table 3 records the incidence of squint.

The incidence of squint among all those drawn for Treatment irrespective of whether treatment was given or not was 19 out of 129 . This is less than the incidence of squint in those drawn for 'no treatment', 28 out of 136 , but the difference is not significant $(\mathrm{p}=0 \cdot 28)$.

\section{Discussion}

The findings in this series of children confirm three observations made previously' ${ }^{19}$ : (1) amblyopia is more predictable than squint; (2) amblyopia is significantly related to meridional hypermetropia at age 1 with a cut off point at $+4.00 \mathrm{D}$ (atropine) or $+3.50 \mathrm{D}$ (cyclopentolate).

Unfortunately, however, it is clear that spectacle correction of hypermetropia or hypermetropic anisometropia from age 1 year has not contributed to the prevention of squint and/or amblyopia. Even after additional occlusion treatment the acuity of the 'treated' group was the same as in the 'untreated' group. It must be said, however, that only onequarter of those who had occlusion had it before age $3 \frac{1}{2}$ years. This was because in many cases visual acuity was not assessed before $31 / 2$. It will never be known whether the final acuity of these children would have been improved by earlier and perhaps more enthusiastic occlusion therapy.

Our previous paper' drew attention to the observation that children who are likely to end up (under current methods of management) with the most severe amblyopia are potentially identifiable because of highly abnormal refractions in $3.7 \%$ of the population at age 1 year. ${ }^{9}$ Better results might be achieved if we knew why this particular project failed. Four possible explanations come to mind. Firstly, occlu- sion was given too late and not enough was given. We think it would not be easy, in practice, to demonstrate that earlier occlusion would be more effective, particularly in those children who do not have additional indicators of severe amblyopia such as constant uniocular squint or considerable hypermetropic anisometropia. It is worth pointing out that amblyopia does not inevitably follow uncorrected hypermetropic anisometropia at age 1 year. Secondly, the known facts about form vision deprivation during a sensitive period may not be relevant to the aetiology of squint and amblyopia in the absence of ocular disease such as congenital cataract. However, if they are relevant, the sensitive period may be effectively over by 1 year, and preventive treatment at this age may have been given too late. Thirdly, it is possible that our traditional basic management (spectacle correction of abnormal refractions, particularly hypermetropia) of squint/amblyopia is wrong. Fourthly, in some instances squint and amblyopia may be due to congenital/familial neuronal abnormalities somewhere along the visual pathway and that these happen to be associated with congenitally determined refractive abnormalities.

The easiest one of these to explore is the possibility of earlier optical correction of hypermetropia, and this is currently being tried from the age of 6 months. We wish to thank the general practitioners and their health visitors for enabling us to study the children under their care. We also thank Mrs L A Neale and Mrs D Maddams for their secretarial help.

\section{References}

1 Ingram RM, Traynar MJ, Walker C, Wilson JM. Screening for refractive errors at age 1: a pilot study. BrJ Ophthalmol 1979; 63: 243-50.

2 Ingram RM. Refraction as a basis for screening children for squint and amblyopia. Br J Ophthalmol 1977; 61: 8-15.

3 Thomson WE. In: Symposium on squint. Trans Ophthalmol Soc UK 1924; 44: 238-52.

4 Chavasse B. In: Symposium on non-paralytic squint. Trans Ophthalmol Soc UK 1932; 52: 348-52.

5 Williamson-Noble FA. In: Symposium on non-paralytic squint. Trans Ophthalmol Soc UK 1932; 52: 359-63.

6 Ingram RM. Refraction of 1-year-old children after atropine cycloplegia. Br J Ophthalmol 1979; 63: 343-7.

7 Ingram RM, Barr A. Refraction of 1-year-old children after cycloplegia with $1 \%$ cyclopentolate: comparison with findings after atropinisation. Br J Ophthalmol 1979; 63: 348-52.

8 Ingram RM, Holland WW, Walker C, Wilson JM, Arnold PE, Dally $\mathrm{S}$. Screening for visual defects in preschoolchildren. $\mathrm{Br} \mathrm{J}$ Ophthalmol (in press).

9 Ingram RM, Walker C, Wilson JM, Arnold PE, Dally S. Prediction of amblyopia and squint by means of refraction at age 1 year. Br J Ophthalmol (in press). 